Article

\title{
'All I ever want to eat is pizza and pasta': Italian food temptations associated with adverse perinatal outcomes
}

Stefania Triunfo*, Roberta Mayer, Michelangela Danza, Antonio Lanzone

Department of 'Scienze della Salute della Donna, del Bambino e di Sanità Pubblica', Fondazione Policlinico Universitario 'A. Gemelli' IRCCS, Università Cattolica del Sacro Cuore, L.go Agostino Gemelli, 8, 00168, Rome, Italy. Roberta.mayer@gmail.com (R.M.), michelangela.danza@policlinicogemelli.it (M.D.), antonio.lanzone@policlinicogemelli.it A.L.).

\footnotetext{
${ }^{*}$ Correspondence: stefania.triunfo@policlinicogemelli.it (S.T)
}

Received: date; Accepted: date; Published: date

Abstract: Growing body of evidence endorse the hypothesis of a protective role played by the inutero environment on a suitable fetal programming, mainly sustained by fitting maternal diet. Our purpose was to assess the linkage between maternal food intake and poor obstetric results, with a special focus on typical Italian food. A cross sectional study including delivering women was designed. A self-reported questionnaire about socio-demographic data, obstetric history, and food frequency intake during pregnancy was administrated. A composite of adverse perinatal outcomes (APO) was constructed. Statistically significant differences were found between APO and control group in smoking habit ( 9.7 vs. $3.2 \%, \mathrm{p}=0.045)$ and BMI at delivery ( $27.9 \pm 4.9$ vs. $26.9 \pm 3.9, \mathrm{p}=0.003$ ). Women complicated by any or more APOs reported increased rates of pasta (5.3 \pm 3.6 vs.4.4 \pm 1.9 times per week, $\mathrm{p}<0.001)$ and pizza $(1.9 \pm 3.4$ vs. $1.1 \pm 0.6$, $\mathrm{p}<0.001)$ intake, with lower consumption of vegetables $(5.4 \pm 3.9$ vs. $7.1 \pm 2.9, \mathrm{p}<0.001)$. By logistic regression analysis and after adjustments for maternal age, ethnicity, SES, maternal BMI at delivery, excessive ingestion of pizza (aOR 1.676, 95\%CI 1.199-2.343, $\mathrm{p}=0.033$ ), but not pasta (aOR 1.077, 95\%CI 0.950-1.211, $\mathrm{p}=0.244$ ), was found associated with APO. Vegetable consumption showed a protective role in reducing APOs (aOR 0.897, 95\%CI $0.818-0.985, \mathrm{p}=0.022)$. Nutrition in pregnancy should minimalize pizza intakes.

Keywords: Fook intake, pizza, pasta, vegetables, pregnancy, adverse perinatal outcome.

\section{Introduction}

An appropriate maternal nutrition is estimated as a significant determinant in the successful pregnancy and complete expression of the fetal growth potential. Up-to-date research highlights that the first 1000 days of life starting from conception up to two years of life represent a window of the opportunities to future health [1]. In line with the Barker's hypothesis [2], focused on the intrauterine developmental origins of human adult disease, investigative and clinical approaches had turned the attention to the influence of a selection of factors, defined as means to understand and prevent the inter-generational inheritance of chronic disease susceptibility [3,4]. 
Currently, the substantial increase in the prevalence of common diseases like hypertension, diabetes, obesity, asthma and atopy, observed over the past decades has directed attention to specific changes in the environment as a possible cause of such an unfavorable switch [5]. Among environmental and modifiable factors, the diet is a crucial influencer of population health. Due to its long-lasting impact, pregnancy is a specifically key period for the programming of future condition [6]. The relevance of the excessive or lacking maternal food intake has been demonstrated related to serious pregnancy outcomes such as preeclampsia, hypertension, prematurity, gestational diabetes, and low birth weight [7-10]. Nevertheless, dietary assessment is complex, mainly due to the difficulty of involving, recording and analyzing a multitude of foods and drinks consumed every day and in varying quantities [11]. As well, maternal diet must be considered as population-specific [12], influenced by socio-cultural factors and depending by food availability.

To the present, different approaches have been developed in order to assess the maternal food intake, including (1) food frequency intake questionnaires (FFQs), addressed on type and frequency of single food or nutrients in isolation; (2) maternal dietary patters (MDPs), characterized by quantities, proportions, variety, or combination of different foods, drinks, and nutrients, and their frequency in consumption; and (3) dietary quality (DQ), as a concept to capture diet as a whole by scoring adherence to guidelines, rating the diversity of food choice in key food groups, or scoring predefined food patterns known to protect or impair health (i.e., Healthy Eating Index, HEI 2010 in United States). Irrespective of the methodological approach chosen, a substantial agreement has been expressed in some maternal dietary habits and poorer obstetric results, recognizing both dangerous and protective maternal dairy products intake [10].

In order to add information to this research topic, we explored the association between appreciated Italian food consumption and pregnancy outcomes by using a FFQ technique in a clinical setting of the Italian capital.

\section{Materials and Methods}

\subsection{Study Population}

Between January to October 2018, a cross sectional study including parturient women within 72 hours of giving birth at the Departments of Obstetrics and Gynecology, Fondazione Policlinico Universitario 'A. Gemelli' IRCCS, Rome, was completed. The hospital is a tertiary referral center with an average of 4,000 deliveries per annum at the study time.

Eligible criteria included maternal age more than 18 years, single pregnancies, residence in the referral area of the hospital, and an adequate understanding of the Italian language. The exclusion criteria were inability to carry out the survey and/or a missed answer percentage more than $50 \%$.

The Research and Ethics Committee of Catholic University approved the research protocol (Institutional Review Board, IRB No. 2017.10/16). A written informed consent was signed by enrolled patients at time of interview.

\subsection{Data Collection}

To elucidate the triad patient-food intake-perinatal outcomes, a multilevel data collection was assessed by using both electronic source information and a target questionnaire. Firstly, maternal demographic characteristics, including age at delivery, ethnicity, socioeconomic status (calculated by including level of study and occupational status), marital status (married/unmarried), and smoking 
habit were collected. Secondly, data pertaining to medical and obstetric history, anthropometric (at pregnancy beginning and at delivery), emerging complications during pregnancy, mode of delivery (vaginal deliver (VD)/caesarean section (CS)), and perinatal outcomes were revised. Thirdly, dietary intake assessed by a validated multi-item semi quantitative FFQ for the 9 months of pregnancy.

\subsection{Dietary Intake Assessment}

The tailored questionnaire was administrated in enrolled patients within 72 hours of giving birth. The completion and return of the questionnaire confirmed the study participation. All women filled the validated FFQ [13]. They were assisted by trained personnel (R.M.). FFQ assesses consumption frequency per week, portion size, and subjective evaluation of food intake. For each food item, including single dietary product (i.e., potatoes, milk, egg) and combined food groups created based on the similarity of nutrient profiles or culinary consumption (i.e., vegetables group), the respondents reported frequency of average consumption. A target focus was addressed on Italian food (i.e., pizza and pasta) and water consumption per day. In order to apply quantitative meaning to the frequency categories, the following numerical values were possible: (a) never, (b) number of times per week or (c) quantity every day.

\subsection{Definition and outcome}

Gestational diabetes was detected based on American Diabetes Association (ADA) criteria [14] (fasting $\geq 95 \mathrm{mg} / \mathrm{dL} ; 1$ hour $\geq 180 \mathrm{mg} / \mathrm{dL} ; 2$ hours $\geq 155 \mathrm{mg} / \mathrm{dL} ; 3$ hours $\geq 140 \mathrm{mg} / \mathrm{dL}$. Hypertensive disorders were defined according to the updated guidelines by ISSHP [15]. Gestational hypertension was defined as the onset of hypertension (systolic $\mathrm{BP} \geq 140 \mathrm{mmHg}$ and/or diastolic $\mathrm{BP} \geq 90 \mathrm{mmHg}$ ) after 20 weeks of gestation which returned to normal within 3 months of delivery, without de novo proteinuria. PE was clinically diagnosed when the novo proteinuria accompanied hypertension. Proteinuria was defined as 24 hours-urine collection with a total protein excretion $\geq 300 \mathrm{mg}$, or $\geq 1 \mathrm{~g} / \mathrm{l}$ in a random urine sample, or a protein/creatinine ratio $\geq 0.3$, without urinary infection. Eclampsia was defined as the development of convulsions and/or unexplained coma during pregnancy or postpartum in patients with signs and symptoms of preeclampsia. The HELLP syndrome was defined by the presence of all three of the following criteria: hemolysis (characteristic peripheral blood smear, total bilirubin $>1,2 \mathrm{mg} / \mathrm{dL}$ and serum lactate dehydrogenase $>600 \mathrm{U} / \mathrm{L}$ ), elevated liver enzymes (serum aspartate aminotransferase $>70 \mathrm{U} / \mathrm{L}$ ), and low platelet count $(<100,000 / \mu \mathrm{L})$. Prematurity was considered in cases of birth before 37 gestational weeks. Grown restriction in newborn was defined in agreement with the latest consensus, including information of birthweight and prenatal feto-maternal Doppler assessment [16].

APO was defined as the presence of maternal or neonatal complication. Adverse outcomes for the women were the following: hypertension and pre-eclampsia, gestational diabetes, need for and length of antenatal hospital stay, antepartum hemorrhage requiring hospitalization, preterm prelabour ruptured membranes, chorioamnionitis, induction of labour, postpartum hemorrhage, perineal trauma (degree III and IV), wound infection, endometritis, length of postnatal hospital stay, thromboembolic disease and maternal death. Adverse outcomes for the offspring were the following: no reassuring fetal status requiring emergency cesarean section, a 5-min Apgar score of $<7$, neonatal metabolic acidosis at birth, defined as $\mathrm{UA} \mathrm{pH} \leq 7.15$ and base excess $>12 \mathrm{mEq} / \mathrm{L}$, admission in 
127

128

129

130

131

132

133

134

135

136

137

138

139

140

141

142

143

144

145

146

147

148

149

150

151

152

153

154

155

neonatal intensive care unit, birth trauma, shoulder dystocia, large for gestational age (LGA), growthrestriction at birth. The composite was defined as any of the above adverse birth complications.

\subsection{Statistical analysis}

Normal distributions were assured by the Shapiro-Wilk test. The Student's t-test for independent samples, the Mann-Whitney-U test, Pearson's chi-square or exact Fisher's tests were used to analyze collected data, as appropriate. Results are presented in means and standard deviations (SD) or number and percentage. Linear regression model was run to examine associations between food intake and adverse perinatal outcomes. Adjustments for maternal age, ethnicity, SES, maternal BMI at starting of the pregnancy and at delivery were performed to calculate adjusted odd ratios (aORs) and 95\% confidence intervals (CIs).

All tests were two-sided, and p-values lower than 0.05 were established statistically significant. IBM SPSS 23.0 (Armonk, NY, USA) and R version 2.15.1 (The R Foundation for Statistical Computing) with package version 1.7.2 software were used for statistical analyses.

\section{Results}

In the period study, 450 delivering women referred for clinical assistance at birth at the Department of Obstetrics and Gynecology, Fondazione Policlinico Universitario 'A. Gemelli' IRCCS in Rome, were enrolled. An agreement in the participation was obtained in 406 patients, with a loss of $9.8 \%(n=44 / 503)$.

\subsection{Population Characteristics}

Presence of one or more complications were identified in 186 (45.8\%) patients (APO group), matched with leaving 220 (34.2\%) women counted in the control group.

Demographic characteristics of the overall study population and subgroups were described in Tab. 1 .

Tab. 1. Demographic characteristics of the study groups, expressed as mean (SD) or $n$ (\%), as appropriate.

\begin{tabular}{lcccc}
\hline Variables & $\begin{array}{c}\text { Overall } \\
\text { population } \\
(\mathbf{n}=\mathbf{4 0 6 )}\end{array}$ & $\begin{array}{c}\text { APO } \\
\text { Group } \\
(\mathbf{n = 1 8 6})\end{array}$ & $\begin{array}{c}\text { Control } \\
\text { Group } \\
(\mathbf{n}=\mathbf{2 2 0})\end{array}$ & p-value \\
\hline Maternal Age & $34.2(4.8)$ & $34.2(4.4)$ & $33.8(5)$ & 0.476 \\
Ethnicity & & & & 0.909 \\
- Caucasian & $385(94.8)$ & $171(91.9)$ & $214(97.3)$ & 0.865 \\
- Latin-American & $9(2.2)$ & $5(2.7)$ & $4(1.8)$ & 0.123 \\
- Afro-Caribbean & $2(0.5)$ & $2(1.1)$ & $0(0)$ & 0.123 \\
- Maghreb & $2(0.5)$ & $2(1.1)$ & $0(0)$ & 0.041 \\
- Asiatic & $8(2)$ & $6(3.2)$ & & 0.352 \\
Level of study & & & $37(27.8)$ & 0.351 \\
\hline
\end{tabular}




$$
\text { - Superior }
$$

Occupational status

- Working

- No-working

SES 1

$$
209 \text { (61.8) }
$$

223 (73.6)

80 (26.4)

22 (5.4)

107(26.4)

209 (51.4)

Marital status

- Married

- Unmarried

Smoking status

$$
\begin{array}{ll}
\text { - } & \text { Smokers } \\
\text { - } \quad \text { Not smokers }
\end{array}
$$

BMI

$$
\begin{aligned}
& 250(61.6) \\
& 116(28.6)
\end{aligned}
$$

$28(6.9)$

91 (58.7)

101 (70.1)

43 (29.9)

$$
7 \text { (5.7) }
$$

89 (64.2)
89 (66.9)

89 (84.8)

26 (25.2)

11 (7.1)

53 (34.2)

91 (58.7)

106 (63.5)

61 (36.5)

7 (3.2)

213 (96.8)
18 (9.7)

168 (90.3)

$\begin{array}{lcc}\text { - Preconception } & 22.9(4.4) & 22.9(4.5) \\ \text { - At starting } & 22.9(4.4) & 23(4.4) \\ \text { - At delivery } & 27.5(4.5) & 27.9(4.9)\end{array}$

APO: adverse perinatal outcomes; ${ }^{2}$ SES: Socio-economic status level, defined as 'low' in presence of the two-following combinations: no study plus no-working status, or primary of level of study plus working status; as 'medium' in presence of the two-following combinations: secondary level of study plus no-working status, or secondary of level of study plus working status; and 'high' in presence of the two-following combinations: superior level of study plus no-working status, or superior of level of study plus working status. BMI: body mass index.

162

Not statistically significant differences were found between APO and control group in mean maternal (34.2 \pm 4.4 vs. $33.8 \pm 5$ years, $\mathrm{p}=0.476)$, level of study, occupational status, SES, and marital status. Only Asian ethnicity showed a significant linkage to APO (3.2 vs. 0.9, p=0.041). Smoking habit ( 9.7 vs. $3.2 \%, p=0.045)$ and BMI at delivery ( $27.9 \pm 4.9$ vs. $26.9 \pm 3.9, p=0.003)$ were statistically different. Medical and obstetric history, perinatal outcomes of the study groups are stated in Tab. 2.

\begin{tabular}{lccc}
\hline & $\begin{array}{c}\text { APO } \\
\text { Group } \\
(\mathbf{n}=\mathbf{1 8 6})\end{array}$ & $\begin{array}{c}\text { Control } \\
\text { Group } \\
(\mathbf{n}=\mathbf{2 2 0})\end{array}$ & p-value \\
\hline Nulliparity & $83(44.6)$ & $52(23.6)$ & 0.034 \\
ART & $12(6.5)$ & $7(3.2)$ & 0.396 \\
GA at delivery & $38.8(1.6)$ & $39.2(1.2)$ & 0.008 \\
Male gender & $96(51.6)$ & $101(45.9)$ & 0.678 \\
AGA neonates & $81(43.5)$ & $220(100)$ & $<0.001$ \\
SGA neonates & $57(30.6)$ & $0(0)$ & $<0.001$ \\
LGA neonates & $48(25.8)$ & $0(0)$ & $<0.001$ \\
\hline
\end{tabular}




\begin{tabular}{lccc}
\hline Intrapartum fever & $26(6.4)$ & $0(0)$ & $<0.001$ \\
Vaginal delivery & $119(63)$ & $201(91.4)$ & $<0.001$ \\
Instrumental vaginal delivery & $10(0.5)$ & $2(0.9)$ & 0.016 \\
Perineal integrity & $14(10.9)$ & $80(39.4)$ & $<0.001$ \\
Spontaneous vaginal lacerations & $77(59.7)$ & $91(44.8)$ & 0.003 \\
Episiotomy & $38(29.5)$ & $32(14.8)$ & $<0.001$ \\
Elective cesarean section & $25(13.4)$ & $17(7.7)$ & $<0.001$ \\
Emergent cesarean section & $32(1.6)$ & $0(0)$ & $<0.001$ \\
NICU admission & $31(1.6)$ & $0(0)$ & $<0.001$ \\
\hline
\end{tabular}

171 APO: adverse perinatal outcomes; ART: Assisted reproductive technology; AGA: appropriate for 172 gestational age; SGA: small for gestational age; LGA: large for gestational age; NICU: neonatal 173 intensive care unit.

174

175 There were no significant differences by ART and gender. Conversely, mode of delivery 176 (instrumental vaginal delivery, elective and emergent CS), categories of AGA, SGA and LGA 177 neonates, spontaneous vaginal lacerations, the necessity of episiotomy, intrapartum fever and 178 necessary neonatal intensive care unit admission were found as statistically significant between two 179 study groups.

180

\subsection{Dietary Intakes}

182 Estimated daily intake of energy, macro and micronutrients during pregnancy are detailed in Tab. 3.

183

184 Tab. 3. Maternal dietary patterns in pregnancy, expressed as mean (SD) or n (\%) per week, as 185 appropriate.

\begin{tabular}{lccc}
\hline \multicolumn{1}{c}{$\begin{array}{c}\text { Nutrients Intake } \\
\text { (per week) }\end{array}$} & $\begin{array}{c}\text { APO } \\
\text { Group } \\
(\mathbf{n}=\mathbf{1 8 6})\end{array}$ & $\begin{array}{c}\text { Control } \\
\text { Group } \\
\text { (n=220) }\end{array}$ & p-value \\
\hline Milk & $6.3(4)$ & $5.7(2.8)$ & 0.112 \\
Cereals & $2.5(3)$ & $2.2(2.8)$ & 0.358 \\
Cake at breakfast & $3.9(3)$ & $3.8(3.2)$ & 0.798 \\
Pasta & $5.3(3.6)$ & $4.4(1.9)$ & 0.044 \\
Rice & $1.8(1.5)$ & $1.7(1.2)$ & 0.500 \\
Meat & $4.2(3.5)$ & $3.9(1.9)$ & 0.489 \\
Fish & $2.2(3.3)$ & $1.9(1.1)$ & 0.078 \\
Eggs & $1.8(1.4)$ & $1.4(0.8)$ & 0.182 \\
Cheese & $3(3.6)$ & $2.9(2.1)$ & 0.838 \\
Vegetable & $5.4(3.9)$ & $7.1(2.9)$ & 0.045 \\
Potatoes & $1.8(1.2)$ & $1.7(1.6)$ & 0.705 \\
Legumes & $2.2(2.8)$ & $2.2(1.4)$ & 0.857 \\
Bread & $5.4(3.8)$ & $5.2(2.2)$ & 0.642 \\
Fruits & $7.2(4.3)$ & $7.1(3.4)$ & 0.863 \\
Cakes & $3.3(3.8)$ & $2.9(2.6)$ & 0.331 \\
Condiments & $6.2(4.2)$ & $6.8(3.6)$ & 0.247 \\
\hline
\end{tabular}




\begin{tabular}{lccl}
\hline Soft Drinks & $2.5(3)$ & $2.7(3.3)$ & 0.679 \\
Alcohol & $0.3(0.8)$ & $0.2(0.7)$ & 0.875 \\
Wine/Beer & $0.1(0.1)$ & $0.1(0.1)$ & 0.901 \\
Cakes & $2(2.6)$ & $2.1(2.4)$ & 0.872 \\
Chocolate & $2.1(3.7)$ & $1.9(1.9)$ & 0.507 \\
Pizza & $1.9(3.4)$ & $1.1(0.6)$ & 0.023 \\
Convivial Lunches & $1.3(1.3)$ & $1.2(0.8)$ & 0.375 \\
Water & $2(0.6)$ & $2.1(0.5)$ & 0.694 \\
\hline
\end{tabular}

186

187

188

189

190

191

192

193

194

195

196

197

198

199

200

APO: adverse perinatal outcomes.

Women complicated by any or more adverse perinatal outcomes reported increased rates of pasta (5.3 \pm 3.6 vs. $4.4 \pm 1.9$ times per week, $\mathrm{p}<0.001)$ and pizza $(1.9 \pm 3.4$ vs. $1.1 \pm 0.6, \mathrm{p}<0.001)$, with lower consumption of vegetables $(5.4 \pm 3.9$ vs. $7.1 \pm 2.9, \mathrm{p}<0.001)$. Similar eating in meat $(4.2 \pm 3.5$ vs. $3.9 \pm 1.9$, $\mathrm{p}=0.489)$, fish $(2.2 \pm 3.3$ vs. $1.9 \pm 1.1, \mathrm{p}=0.078)$, fruits $(7.2 \pm 4.3$ vs. $7.1 \pm 3.4, \mathrm{p}=0.863)$, and legumes $(2.2 \pm 2.8$ vs. $2.2 \pm 1.4, \mathrm{p}=0.857)$ in both groups. Any statistical differences were found between APO and control group in other nutrients intake, including water drinking.

By logistic regression analysis and after adjustments for maternal age, ethnicity, SES, maternal BMI at delivery, excessive ingestion of pizza (aOR 1.676, 95\%CI 1.199-2.343, p=0.033), but not pasta (aOR 1.077, 95\%CI 0.950-1.211, $\mathrm{p}=0.244$ ), was found associated with APO (Tab. 4). Vegetable consumption showed a protective role in reducing APO (aOR 0.897, 95\%CI 0.818-0.985, p=0.022). All other nutrients were excluded by the model.

Tab. 4. Associations of maternal dietary food intake in pregnancy with APO

\begin{tabular}{lcccc}
\hline & \multicolumn{3}{c}{ Model $^{\mathbf{a}}$} \\
\cline { 2 - 5 } & B & SE & OR [95\% CI] & p-value \\
Pizza & 0.517 & 0.171 & $1.676[1.199-2.343]$ & 0.033 \\
Pasta & 0.075 & 0.064 & $1.077[0.950-1.211]$ & 0.244 \\
Vegetable & -0.108 & 0.047 & $0.897[0.818-0.985]$ & 0.022 \\
\hline
\end{tabular}

SE: standard error; OR: odd ratio; CI: confidence interval.

aAdjusted for maternal age, ethnicity, SES, maternal BMI at delivery.

\section{Discussion}

\subsection{Main findings}

As first study focused on selected and appreciated Italian food intake during pregnancy, we assessed the unsafe association between pizza consumption and pregnancy, demonstrating increased rates of APO in those pregnant women more inclined to eat it. Although statistically significant in univariate analysis, eating pasta every day did not increase the risk of APO in a multivariate model. Finally, we endorsed the protective role played by vegetable ingestion to successful obstetric results.

\subsection{Interpretation in light of other evidence}

During pregnancy the adequate nutritional status determines the favorable outcomes for the mother-baby dyad. This issue is a well-recognized starting point in all recommendations and guidelines delivered by national and international societies and health care systems $[12,13,17]$. Programs of diary diet and variations across the trimesters of pregnancy underlines benefits from heathy nutrition, suggesting type and dose of food and recommending daily requirement of 
macronutrients and micronutrients. Specific recommendations are developed in according to both the eating tradition and nutrition status of the population [13,17]. In the undernourished population, balanced energy and protein intake are recommended to prevent maternal and fetal complications. Supplementation of vitamin A, calcium or vitamin D is strongly suggested in areas with deficiency, or low-calcium intake, or low sun exposure [18]. Avoiding of caffeine and alcohol is suggested for women with high and moderate consumption, respectively. In Italy, a recent consensus advises both energy input and protein intake during pregnancy with specific amounts mainly recommended in the selected periods [13]. In general, the emphasis is put on the protein and fat composition, iron supplementation, as well as iodine and calcium adequate provision.

Apart from the recommendations, there is substantial body of research output concerning specific aspects of maternal nutrition and APO. A recurrent approach includes the identification of maternal dietary patterns using FFQs, able to obtain a comprehensive account of dietary information, although with inevitable misclassifications in dietary intake or limitations of recall bias. Nevertheless, the inclusion of different type of food in an identical named maternal dietary pattern might leave contrasting results across studies [19]. This might be elucidated by comparing the several definitions of Mediterranean Diet, Mediterranean-type diet, Mediterranean Diet Index and Mediterranean Diet Score or the list of food in the 'Western pattern'. Of interest, from these investigations, associations between selected dietary patterns and the development of adverse obstetric outcomes have been demonstrated, including the more frequent such as fetal growth disorders, gestational diabetes, hypertensive disorders, prematurity, as revised by Chen and co-workers recently [19].

Anyway, the analysis according to dietary patterns in pregnancy is lacking in ascertaining the role of certain food not directly attributable to one or more patterns or those typical in specific areas or culture. Indeed, foods might be consumed in isolation and dietary patterns cannot be useful to assess the whole diet consumed. As universal food, pizza and pasta represent a real temptation also in pregnancy. While pasta does not seem to be dangerous, daily intake of pizza has been found associated with APO in our representative setting of the Italian mixed population in pregnancy. It could be elucidated if we assumed pizza as a substitutive role of the bread, mainly if it is bakes without sweet tomato sauce and salty mozzarella cheese. While there are exceptions, pizza most is high in calories, sugar and sodium. Additionally, not always ingredients and preparation methods are listed. In contrast to frozen pizzas, freshly made ones often contain healthier ingredients than the more processed ones sold in convenience stores and fast-food restaurants. Most pizzerias make their dough from scratch using simple ingredients like olive oil and wheat flour and cooking in woodfired oven rather than in electric one. Depending on the restaurant, some use homemade sauces with no added sugar, fresh cheeses and other healthy toppings. However, in our opinion, no matter if the choose is frozen or fresh pizza, piling on extra toppings can make it unhealthy. In a recent study among Tehranian women, Lamyian et al. reported that the pre-pregnancy consumption of total fast foods including pizza was not found to have undesirable effects in the prevalence of gestational diabetes [20]. Likewise, in a cross-sectional study, no association was found between the unhealthy retail food outlets such as fast food, pizza, bodegas, bakeries, convenience, candy/nut and meat stores in the neighborhood and gestational diabetes [21]. Conversely, in other investigations, higher consumption of fast foods was associated with an increased risk of APO [22,23]. Our findings are in general agreement with these studies, since the components of pizza might include a high fat content and saturated fatty acids. Indeed, previous reports show that high intakes of saturated fatty acids suppress activity of pancreatic Mgat4a-encoded GlcNAcT-IV glycosyltransferase [24], increase both body weight and blood glucose, and induce placental oxidative stress and vascular dysregulation, frequent in some conditions such as hypertensive disorders, abnormal fetal growth and gestational diabetes. Following the explication of negative impact of pizza intake, we could speculate that the energy density of the pizza, or more in general of fast food, can disturb the regulation of appetite and result in a significant reduction in sensory-specific satiety. This might play the role of a significant hedonic inhibitor of energy intake [25], leading to overweight, obesity, and insulin resistance, and therefore APO [26-29]. 
As a modifiable factor, dietary patterns may be more applicable to clinical and pregnant health interventions. Outstandingly, the diets with higher intake of fruits, vegetables, legumes and fish have positive pregnancy outcomes in general $[13,17]$. Several studies have found that vegetable intake might reduce APOs. Among all, the INMA study [30] reported a beneficial effect on fetal growth attributed by the authors to the high content of antioxidants and folic acid in vegetables. From Denmark [31] and India [32] similar findings were renowned in additional reports. Also in our cohort, their intake was found as a protective factor against APO when assumed regularly across pregnancy trimesters.

In light of these evidence, diet represent a key area for intervention in pregnant women. Recommendations proposed by different authorities are based on the solid medical literature. Nevertheless, differences-population specific in food intake, related to eating customs and tradition, require extra advices among pregnant women. As consequence, during each prenatal care visit, medical professionals must address multiple nutritional issues. To improve the quality of diet for pregnant women include the participation of a multidisciplinary team in providing educational strategies, support groups, and education about diet benefits and safety for both pregnant women and neonates.

This study has some strengths and limitations. It is a prospective study designed to assess the association between carefully chosen Italian food and pregnancy outcomes. A well-defined compound of APO has been constructed in order to clarify the causative linkage malnutritionunsuccessful pregnancy. Although the study design was cross-sectional, women were asked about food intake during pregnancy in postpartum, which could lead to the "recall bias".

\section{Conclusions}

An unsafe association between pizza consumption and pregnancy has been demonstrated due to increased rates of APO in those pregnant women more inclined to eat it, while regular vegetable ingestion contributes to successful obstetric results. For the reason that nutrition in pregnancy is a pivotal issue in preventive medicine, endorsements in safe food intake are warranted, with special attention on cultural aspects and geographic areas.

Author Contributions: Conceptualization, S.T, R.M. and M.D.; methodology, S.T, R.M.; formal analysis, S.T.; data curation, R.M.; writing - original draft preparation, S.T.; writing - review and editing, S.T., R.M., M.D. and A.L.; project administration, S.T.

Funding: None

Acknowledgments: None

Conflicts of Interest: The authors declare no conflict of interest

\section{References}

1. Stephenson, J.; Heslehurst, N.; Hall, J.; Schoenaker, D.A.J.M.; Hutchinson, J., Cade, J.E.; Poston, L.; Barrett, G., Crozier, S.R.; Barker, M.; Kumaran, K.; Yajnik, C.S.; Baird, J.; Mishra, G.D. Before the beginning: nutrition and lifestyle in the preconception period and its importance for future health. Lancet 2018, 5 3911830-1841.

2. Barker, D.J. In utero programming of chronic disease. Clin Sci (Lond) 1998, 95 115-128.

3. Moore, L.E. Recurrent risk of adverse pregnancy outcome. Obstet Gynecol Clin North Am 200835459 471.

4. Park, K.; Wei, J.; Minissian, M.; Bairey Merz, C.N.; Pepine, C.J. Adverse Pregnancy Conditions, Infertility, and Future Cardiovascular Risk: Implications for Mother and Child. Cardiovasc Drugs Ther $201529391-401$. 
5. Li, A.M. Ecological determinants of health: food and environment on human health. Environ Sci Pollut Res Int 201724 9002-9015.

6. Danielewicz, H.; Myszczyszyn, G.; Dębińska, A.; Myszkal, A.; Boznański, A.; Hirnle, L. Diet in pregnancy-more than food. Eur J Pediatr 2017, 176 1573-1579.

7. Ramakrishnan, U., Grant, F.; Goldenberg, T.; Zongrone, A.; Martorell, R. Effect of women's nutrition before and during early pregnancy on maternal and infant outcomes: a systematic review. Paediatr Perinat Epidemiol 201226 285-301.

8. Mousa, A.; Naqash, A.; Lim, S. Macronutrient and Micronutrient Intake during Pregnancy: An Overview of Recent Evidence. Nutrients. 2019, 2011.

9. Martin, J.C.; Zhou, S.J., Flynn, A.C., Malek, L.; Greco, R., Moran L. The Assessment of Diet Quality and Its Effects on Health Outcomes Pre-pregnancy and during Pregnancy. Semin Reprod Med 2016 34 83-92.

10. Doyle, I.M.; Borrmann, B.; Grosser, A.; Razum, O.; Spallek, J.Determinants of dietary patterns and diet quality during pregnancy: a systematic review with narrative synthesis. Public Health Nutr 201720 1009-1028.

11. Trichopoulos, D.; Lagiou, P. Dietary patterns and mortality. Br J Nutr 200185 133-134.

12. Marangoni F, Cetin I, Verduci E, Canzone G, Giovannini M, Scollo P, Corsello G, Poli A. Maternal Diet and Nutrient Requirements in Pregnancy and Breastfeeding. An Italian Consensus Document. Nutrients. 2016 Oct 14;8(10). pii: E629.

13. SIGO. Nutrizione in gravidanza e durante l'allattamento. 2018. https://www.sigo.it/wpcontent/uploads/2018/06/lg_nutrizioneingravidanza.pdf.

14. American Diabetes Association. Standards of medical care in diabetes. Diabetes Care. 2016 39 S1-S106.

15. International Society for the Study of Hypertension in Pregnancy. The classification, diagnosis and management of the hypertensive. disorders of pregnancy: A revised statement from the ISSHP. Pregnancy Hypertension: An International Journal of Women's Cardiovascular Health. 2014 4 97-104.

16. Beune IM, Bloomfield FH, Ganzevoort W, Embleton ND, Rozance PJ, van Wassenaer-Leemhuis AG, Wynia K, Gordijn SJ. Consensus Based Definition of Growth Restriction in the Newborn. J Pediatr. 2018 $19671-76$.

17. ACOG. Nutrition During Pregnancy. FAQ001 2018. https://www.acog.org/Patients/FAQs/NutritionDuring-Pregnancy?IsMobileSet=false

18. Triunfo S.; Lanzone A.; Lindqvist P.G. Low maternal circulating levels of vitamin D as potential determinant in the development of gestational diabetes mellitus. Endocrinol Invest 201740 1049-1059.

19. Chen X.; Zhao D.; Mao X.; Xia Y.; Baker P.N.; Zhang H. Maternal Dietary Patterns and Pregnancy Outcome. Nutrients 201678.

20. Lamyian M, Hosseinpour-Niazi S Mirmiran P, Moghaddam Banaem L, Goshtasebi A, Azizi F. PrePregnancy Fast Food Consumption Is Associated with Gestational Diabetes Mellitus among Tehranian Women. Nutrients. 201719.

21. Janevic, T.; Borrell, L.N.; Savitz, D.A.; Herring, A.H.; Rundle, A. Neighbourhood food environment and gestational diabetes in New York City. Paediatr. Perinat. Epidemiol 201024 249-254.

22. Bowers, K.; Tobias, D.K.; Yeung, E.; Hu, F.B.; Zhang, C. A prospective study of prepregnancy dietary fat intake and risk of gestational diabetes. Am. J. Clin. Nutr. 2012, 95, 446-453.

23. Park, S.; Kim, M.Y.; Baik, S.H.; Woo, J.T.; Kwon, Y.J.; Daily, J.W.; Park, Y.M.; Yang, J.H.; Kim, S.H. Gestational diabetes is associated with high energy and saturated fat intakes and with low plasma visfatin and adiponectin levels independent of prepregnancy BMI. Eur. J. Clin. Nutr. 201367 196-201.

24. Ohtsubo, K.; Takamatsu, S.; Minowa, MT.; Yoshida, A.; Takeuchi, M.; Marth, J.D. Dietary and genetic control of glucose transporter 2 glycosylation promotes insulin secretion in suppressing diabetes. Cell 2005123 1307-1321.

25. Tey, S.L.; Brown, R.C.; Gray, A.R.; Chisholm, A.W.; Delahunty, C.M. Long-term consumption of high energy-dense snack foods on sensory-specific satiety and intake. Am. J. Clin. Nutr. $2012951038-1047$.

26. Pereira, M.A.; Kartashov, A.I.; Ebbeling, C.B.; Van Horn, L.; Slattery, M.L.; Jacobs, D.R., Jr.; Ludwig, D.S. Fast-food habits, weight gain, and insulin resistance (the CARDIA study): 15-year prospective analysis. Lancet $200536536-42$.

27. Triunfo S.; Lanzone A. Impact of overweight and obesity on obstetric outcomes. J Endocrinol Invest 2014 37 323-9. 
368

369

370

371

372

373

374

375

376

377

378

379

380

381

382

383

28. Triunfo S.; Lanzone A. Impact of maternal under nutrition on obstetric outcomes. J Endocrinol Invest 201538 31-8.

Martin C.L.; Sotres-Alvarez D.; Siega-Riz A.M. Maternal Dietary Patterns during the Second Trimester Are Associated with Preterm Birth. J Nutr 2015145 1857-1864.

29. Ramón R.; Ballester F.; Iñiguez C.; Rebagliato M.; Murcia M.; Esplugues A. Vegetable but not fruit intake during pregnancy is associated with newborn anthropometric measures. J Nutr 2009139 561567.

30. Mikkelsen T.B.; Osler M.; Orozova-Bekkevold I.; Knudsen V.K.; Olsen S.F. Association between fruit and vegetable consumption and birth weight: a prospective study among 43,585 Danish women. Scand J Public Health 200634 616-622.

31. Rao S.; Yajnik C.S.; Kanade A.; Fall C.H.; Margetts B.M.; Jackson A.A. Intake of micronutrient-rich foods in rural Indian mothers is associated with the size of their babies at birth: Pune maternal nutrition study. J Nutr 2001131 1217-1224. 